\title{
Orotidylate Decarboxylase Inhibitor
}

National Cancer Institute

\section{Source}

National Cancer Institute. Orotidylate Decarboxylase Inhibitor. NCI Thesaurus. Code C2170.

Any substance that inhibits orotidine 5'-phosphate decarboxylase (orotidylate decarboxylase), an enzyme that catalyzes the decarboxylation of orotidine monophosphate to form uridine monophosphate and is required for the de novo synthesis of uridine and pyrimidines. Inhibition of orotidine 5'-phosphate decarboxylase inhibits DNA, RNA, and protein synthesis. 